\title{
Advantages of gross total resection in patients with astrocytoma: A population-based study
}

\author{
HUA MAO $^{1^{*}}$, XIANGUO LI $^{1 *}$ and WEIPU MAO ${ }^{2 *}$ \\ ${ }^{1}$ Department of Neurosurgery, Jingzhou Central Hospital, The Second Clinical Medical College of Yangtze University, \\ Jingzhou, Hubei 434020; ${ }^{2}$ Department of General Practice, Shanghai Tenth People's Hospital, \\ Tongji University School of Medicine, Shanghai 200072, P.R. China
}

Received July 22, 2019; Accepted January 24, 2020

DOI: $10.3892 / \mathrm{ol} .2020 .11514$

\begin{abstract}
The present study aimed to investigate the association between surgical methods and survival outcomes in patients with astrocytoma. Patients diagnosed with astrocytoma between January 2004 and December 2015 were identified using the Surveillance, Epidemiology and End Results database. Kaplan-Meier curves and Cox regression were used to analyze the effects of surgical methods on overall survival (OS) and cancer-specific survival (CSS). Among 42,224 eligible patients with astrocytoma, 11,427 (27.1\%) patients did not receive surgery, 7,661 (18.1\%) received excisional biopsy (EB), 5,520 (13.1\%) received a subtotal resection (STR), 6,037 (14.3\%) received a gross resection (GR), 5,314 $(12.6 \%)$ received a partial resection (PR) and 6,265 (14.8\%) received a gross total resection (GTR). Patients who underwent GR had the longest survival time (17.00 months). However, over time, the proportion of patients who underwent STR or GR increased, whereas the proportion of patients who did not undergo surgery, PR or GTR decreased. Furthermore, surgical method was an independent prognostic factor for OS and CSS for the patients with astrocytoma. Multivariate Cox regression showed that GTR was associated with the more favorable OS [hazard ratio (HR), 0.80; 95\% confidence interval (CI), 0.77-0.83; $\mathrm{P}<0.001]$ and CSS (HR, 0.80; 95\% CI, 0.77-0.83; $\mathrm{P}<0.001)$ times compared with EB. Moreover, similar results were observed in subgroup analyses based on summary stage and grade. In the present study, it was demonstrated that GTR was one of the effective surgical methods for improved OS and CSS time in patients with astrocytoma. However, among the
\end{abstract}

Correspondence to: Dr Hua Mao, Department of Neurosurgery, Jingzhou Central Hospital, The Second Clinical Medical College of Yangtze University, 1 Renmin Road, Jingzhou, Hubei 434020, P.R. China

E-mail: san33mao@126.com

${ }^{*}$ Contributed equally

Key words: astrocytoma, gross total resection, survival outcomes, Surveillance, Epidemiology and End Results
American astrocytoma population, the proportion of patients who underwent GTR decreased. It is necessary to further advocate for the efficacy of GTR.

\section{Introduction}

A glioma is a tumour produced by glial cells and is the most common primary malignant tumour in the central nervous system, accounting for $\sim 30 \%$ of the tumours in this region $(1,2)$. Astrocytoma is one of the most aggressive gliomas, with a poor prognosis $(3,4)$. Astrocytomas can be found in various parts of the central nervous system and are a common neuroepithelial tumour that can occur in individuals of all ages (5). The average survival time ranges from 17 weeks to 3 years (6). The tumours develop slowly and progressively, and epilepsy is often the first symptom. Approximately $50 \%$ of patients experience the onset of epilepsy and the majority of patients experience headaches, psychomotor muscle weakness, vomiting and an obvious disturbance of consciousness (7).

According to the 2016 World Health Organization (WHO) classification, astrocytoma can be divided into four grades (I-IV) based on its histological and morphological characteristics (8): Low-grade astrocytomas are classified as grades I and II, and high-grade astrocytomas are classified as grades III and IV. The prognosis of high-grade astrocytoma is very poor and the average survival time is only $0.6-0.7$ years (9).

The growth pattern of astrocytomas in the brain consists of invasive or local invasive growth, and as the invasiveness increases, the tumour grade increases and the survival rate decreases (10). At present, the conventional treatment is primarily surgical resection supplemented by radiotherapy and chemotherapy (11). There are a number of surgical methods for astrocytoma, including excisional biopsy (EB), subtotal resection (STR), gross resection (GR), partial resection (PR) and gross total resection (GTR). Although the efficacy of conventional treatment has made some progress in recent years, the prognosis for astrocytoma patients is still poor, which is primarily associated with incomplete resection, recurrence and radiotherapy and chemotherapy resistance after surgical resection (12-14). Therefore, it is important to find an optimal treatment for patients with astrocytoma.

The purpose of the present study was to use the Surveillance, Epidemiology and End Results (SEER) database 
to characterize the different therapies for patients with astrocytoma at a population level and the recommendations for treatment use.

\section{Patients and methods}

Data source and patients. The SEER database includes $\sim 28 \%$ of the US population and collects demographic information, primary tumour location, tumour grade, tumour stage, treatment method and survival time data for patients with cancer (15). The National Cancer Institute SEER*Stat software [version 8.3.5; SEER 18 Regs Custom Data (with additional treatment field), Nov 2017 Sub (1973-2015 varying) database] was used to identify 46,717 patients diagnosed with astrocytoma between January 2004 and December 2015. Histological ICD-O-3 codes (The 3rd edition of The International Classification of Diseases for Oncology) (16) were used to select the following subtypes: Subependymal giant cell astrocytoma, malignant (ICD-O-3 code 9384/3); astrocytoma, NOS (ICD-O-3 code 9400/3); astrocytoma, anaplastic (ICD-O-3 code 9401/3); protoplasmic astrocytoma (ICD-O-3 code 9410/3); gemistocytic astrocytoma (ICD-O-3 code 9411/3); fibrillary astrocytoma (ICD-O-3 code 9420/3); pilocytic astrocytoma, malignant (ICD-O-3 code 9421/3); pleomorphic xanthoastrocytoma (ICD-O-3 code 9424/3); glioblastoma, NOS (ICD-O-3 code 9440/3); giant cell glioblastoma (ICD-O-3 code 9441/3); and gliosarcoma (ICD-O-3 code $9442 / 3)$.

The exclusion criteria in the present study were: i) Unknown survival time $(\mathrm{n}=206)$; ii) unknown household income $(n=2)$; iii) age $<18$ years $(n=3,597)$; and surgical code not $00,20,21,30,40$ or 55 ( $n=688$; https://seer.cancer. gov/manuals/2018/AppendixC/Surgery_Codes_Brain_2018.pdf). Ultimately, a total of 42,224 eligible patients diagnosed with astrocytoma.

There were several methods used to confirm the diagnosis of patients in the SEER database, such as histological diagnosis and radiography. Overall, $90.7 \%$ of patients were confirmed by positive histological diagnosis and $7.8 \%$ by radiography (Fig. S1).

Study variables. The study variables in the present study included age at diagnosis, year of diagnosis, sex, ethnicity, marital status, urban-rural residence, household income, summary stage, and surgical, radiotherapy and chemotherapy information. According to the surgical code, patients were divided into four groups: No surgery (code 00), EB (code 20), STR (code 21), GR (code 30), PR (code 40) and GTR (code 55) (17). Astrocytomas were divided into four groups according to the 2016 WHO classification: Grade I, grade II, grade III and grade IV (8). Demographic and clinicopathological characteristics included age at diagnosis (18-40, 41-60, 61-80 and >80 years), sex (male and female), ethnicity (white, black and other), marital status (married, unmarried and unknown), urban-rural residence (metropolitan and non-metropolitan), summary stage (localized, regional, distant and unstaged/unknown), radiotherapy (yes or no) and chemotherapy (yes or no). Household income was divided into three groups: Low-income group $(<4,219)$, middle-income group $(4,219-5,191)$ and high-income group $(>5,191)$. Overall survival
(OS) and cancer-specific survival (CSS) were the primary endpoints of the present study.

Statistical analysis. The OS time corresponded to the length of time from the date of diagnosis to the death from any cause or the date on which data were censored. When analyzing CSS, mortality cases associated with other causes were excluded. SPSS version 20.0 (IBM Corp.) was used for all statistical analyses. $\chi^{2}$ tests were used to analyze factors associated with the surgical methods. Kaplan-Meier curve analyses and the log-rank test were used to analyze the OS and CSS times of patients with regard to different surgical methods and other variables. Multivariate Cox regression was used to analyze factors associated with OS and CSS. $\mathrm{P}<0.05$ was considered to indicate a statistically significant difference

\section{Results}

Demographic and clinicopathological characteristics of the astrocytoma. A total of 42,224 eligible astrocytoma patients from between January 2004 and 2015 December were included in the present study cohort. Among them, 11,427 (27.1\%) patients did not receive surgery, 7,661 (18.1\%) received EB, 5,520 (13.1\%) received STR, 6,037 (14.3\%) received GR, $5,314(12.6 \%)$ received PR and 6,265 (14.8\%) received GTR (Fig. 1A). Table I shows the demographic and clinicopathological characteristics of patients with astrocytoma and the association between surgical method and each variable as analyzed by the $\chi^{2}$ test. $\chi^{2}$ tests showed that age of diagnosis, year of diagnosis, sex, ethnicity, marital status, urban-rural residence, household income, summary stage, radiotherapy and chemotherapy information were all associated factors (all $\mathrm{P}<0.001$ ). Among all 42,224 patients, over time, the number of patients diagnosed with astrocytoma increased. The majority of patients were white [37,462 $(88.7 \%)$; Table I], between 41-80 years old [33,344 (79.0\%)], had localized disease [33,090 (78.4\%); Fig. 2A] and were WHO grade IV [32,876 (77.9\%); Fig. 2B].

Among all 42,224 patients, the proportion of patients who underwent STR or GR increased between 2004 and 2015 [43/3,281 patients $(1.3 \%)$ vs. $957 / 3,871(24.7 \%), \mathrm{P}<0.001$; and $50 / 3,281$ patients $(1.5 \%)$ vs. $1,214 / 3,871$ (31.4\%), $\mathrm{P}<0.001$, respectively]. However, the proportion of patients who did not undergo surgery, PR or GTR decreased between 2004 and 2015 [1,000/3,281 patients (30.5\%) vs. 932/3,871 (24.1\%), $\mathrm{P}<0.001$; $778 / 3,281$ patients $(23.7 \%)$ vs. $57 / 3,871(1.5 \%), \mathrm{P}<0.001$; and $1,020 / 3,281$ patients $(31.1 \%)$ vs. $104 / 3,871(2.7 \%), \mathrm{P}<0.001]$ (Fig. 3).

Subgroup analysis for evaluating the proportion of different surgical methods based on SEER stage and grade. The proportion of different surgical methods based on summary stage and grade were evaluated. As shown in Table I and Fig. 1B-D, compared with patients with regional and distant astrocytoma, patients with localized astrocytoma were more likely to undergo GR (16.2 vs. 7.4 or $8.6 \%)$ and GTR (16.9 vs. 7.5 or $8.2 \%$ ). In addition, patients with WHO grade I and IV were more likely to undergo GR (18.9 and 14.8 vs. 11.1 and $12.6 \% ; \mathrm{P}<0.001)$ and GTR (23.5 and 15.5 vs. 12.1 and 
A

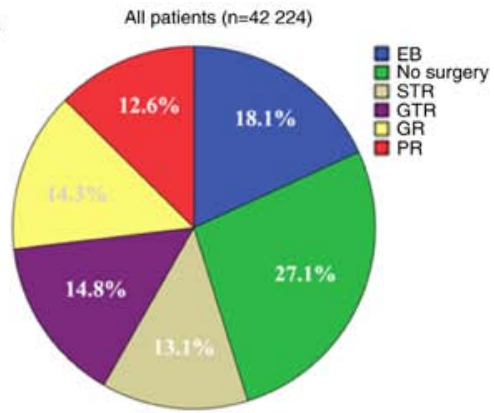

C

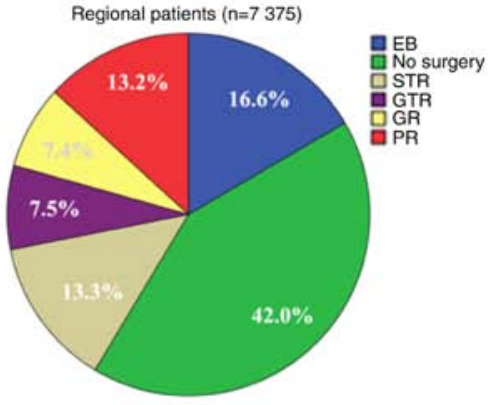

E

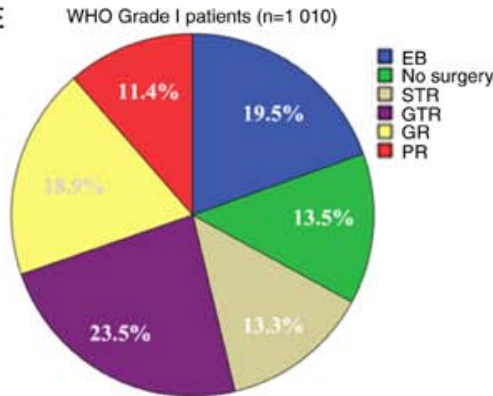

G

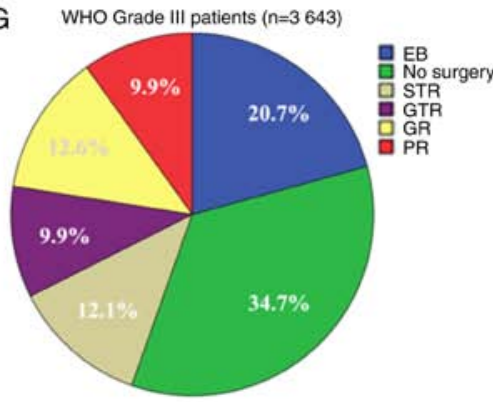

B

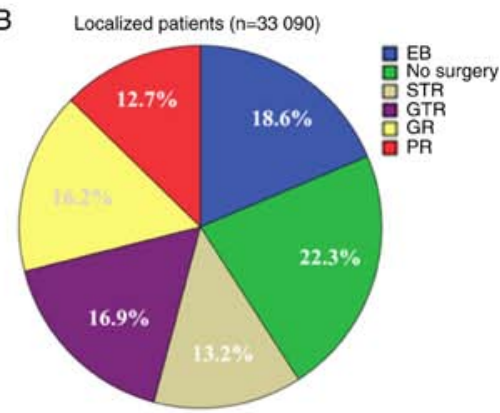

D

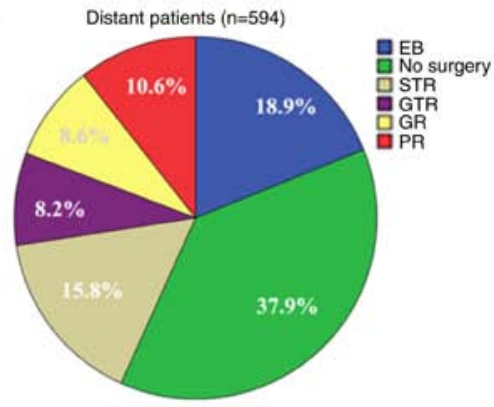

F

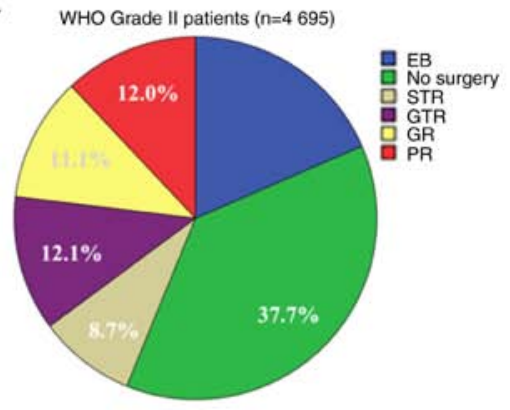

H

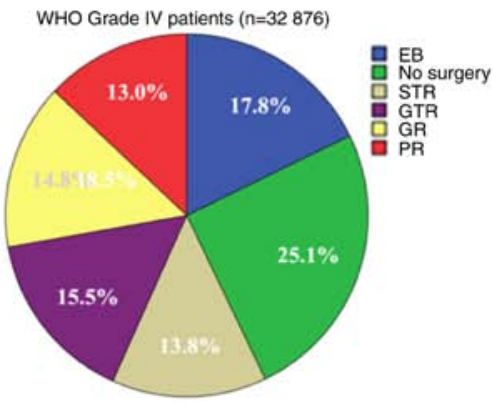

Figure 1. Number and proportion of surgical methods performed on patients with astrocytoma with different summary statuses between 2004 and 2015 . (A) All patients, $n=42,224$. (B) Localized patients, $n=33,090$. (C) Regional patients, $n=7,375$. (D) Distant patients, $n=594$. (E) WHO grade I (7) patients, $\mathrm{n}=1,010$. (F) WHO grade II patients, $\mathrm{n}=4,695$. (G) WHO grade III patients, $\mathrm{n}=3,643$. (H) WHO grade IV patients, $\mathrm{n}=32,876$. WHO, World Health Organization; EB, excision biopsy; STR, subtotal resection; GR, gross resection; PR, partial resection; GTR, gross total resection.

9.9\%; P<0.001) compared with WHO grade II and III (Table I; Fig. 1E-H).

Effects of different variables on OS and CSS in patients with astrocytoma. Kaplan-Meier curves were constructed to analyze the influence of clinical factors on the OS and CSS of patients with astrocytoma (Table II). Kaplan-Meier analysis showed that age at diagnosis, ethnicity, marital status, urban-rural residence, household income, summary stage, WHO grade, and surgical, radiotherapy and chemotherapy information were significantly associated with OS and CSS (all $\mathrm{P}<0.05)$. Patients who underwent GR or GTR had longer OS median survival times (MSTs) (17.00 and 15.00 months) and higher CSS MST (19.00 and 17.00 months) compared with those in the other surgical groups (Fig. 4A and B).

Identification of prognostic factors for patients with astrocytoma. Multivariate Cox regression was used to analyze the factors associated with OS and CSS in patients with astrocytoma (Table III). After adjusting for age at diagnosis, ethnicity, marital status, urban-rural residence, household income, summary stage, WHO grade, and surgical, radiotherapy 


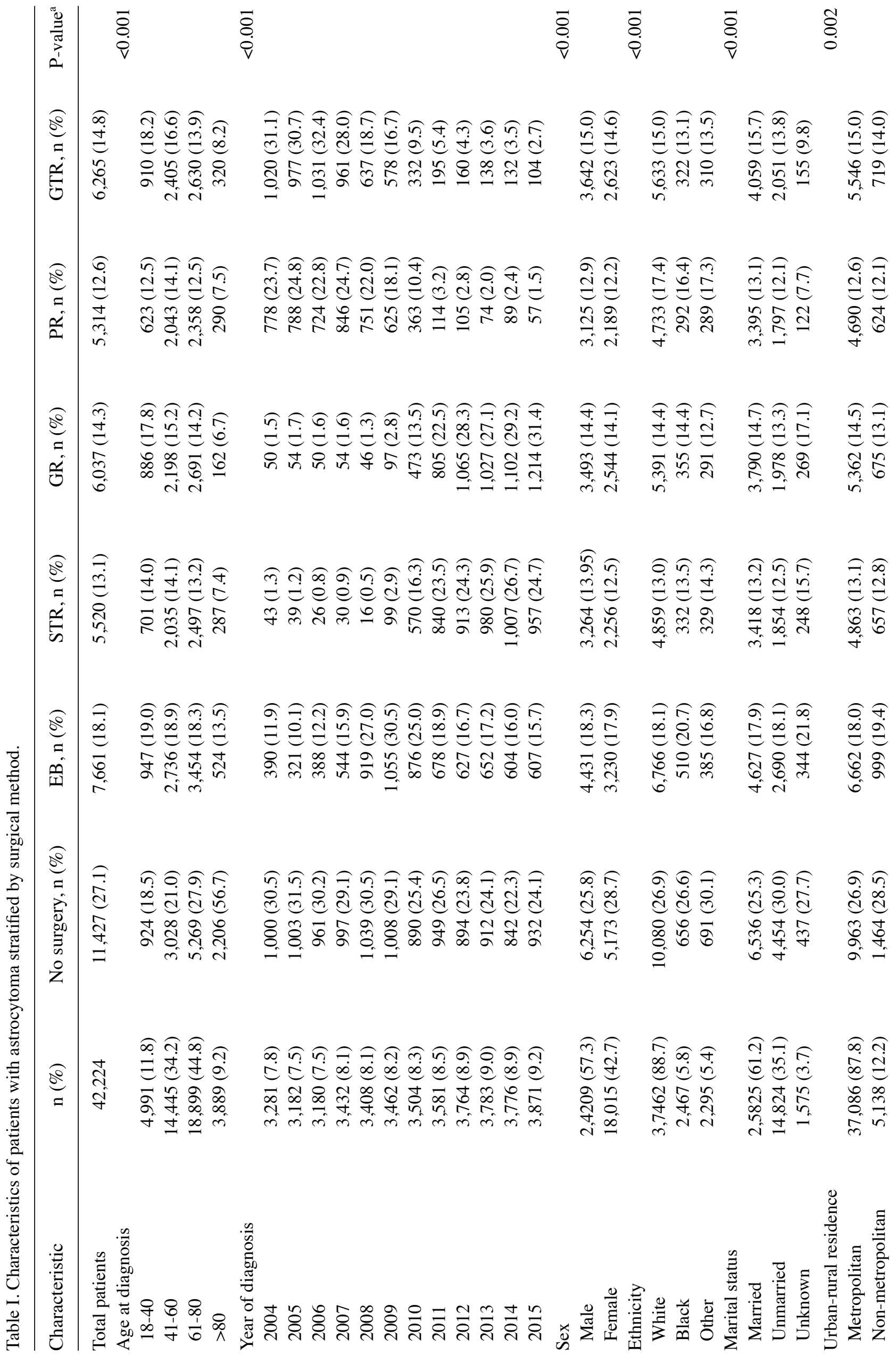




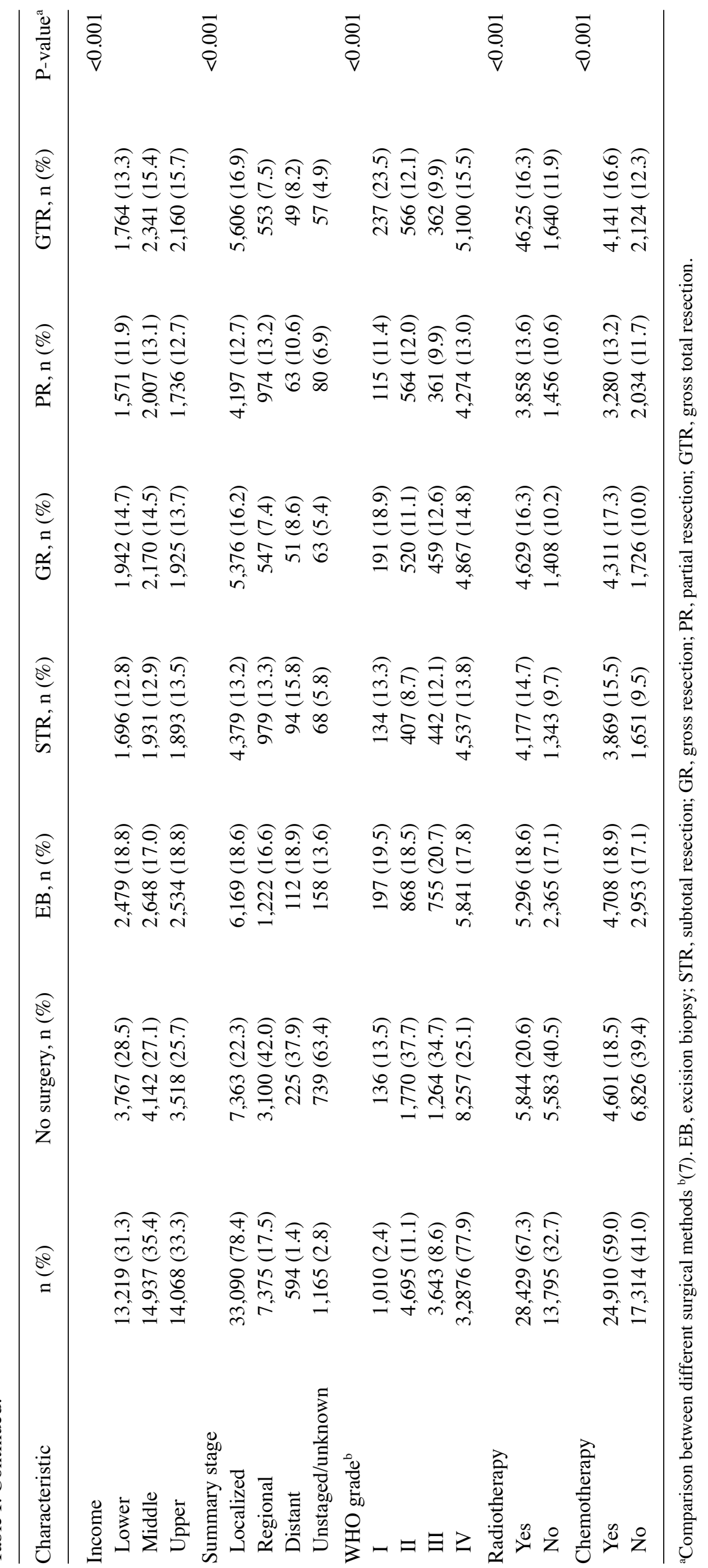


A

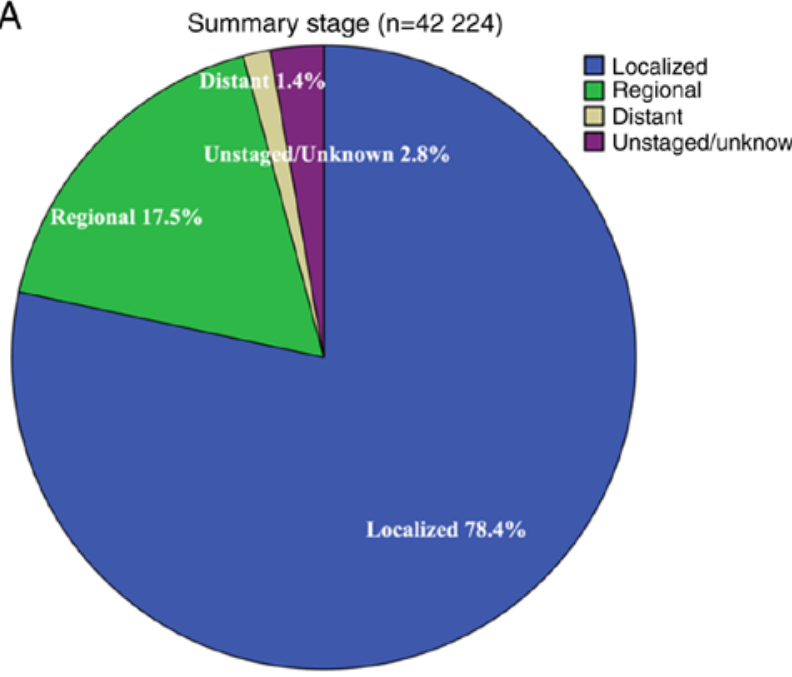

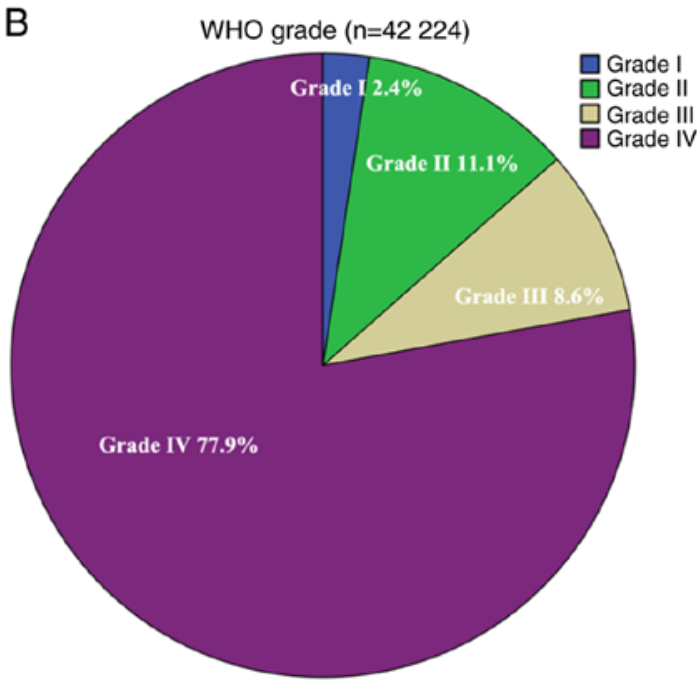

Figure 2. Number and proportion of different summary stages and WHO grades (7) between 2004 and 2015. (A) Different summary stages, $n=42,224$. (B) Different WHO grades, $\mathrm{n}=42,224$. WHO, World Health Organization.

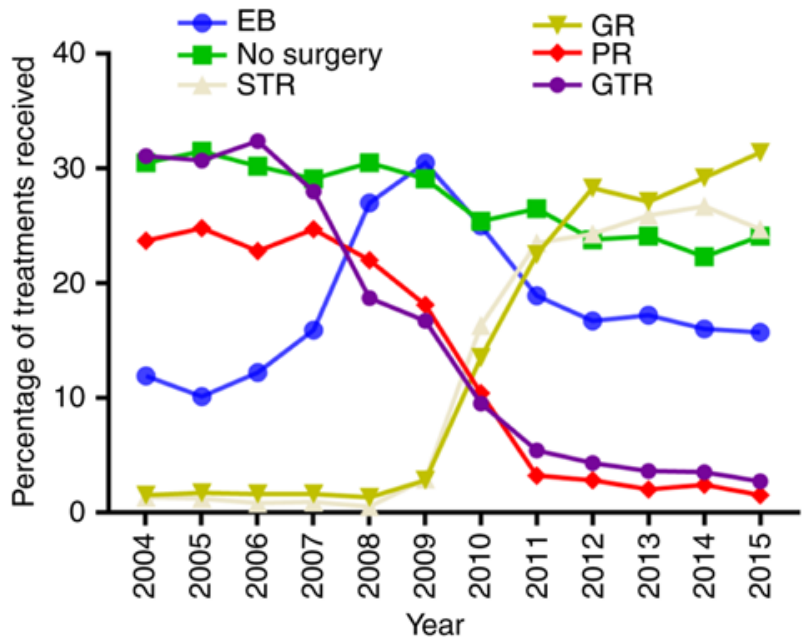

Figure 3. Annual change in the proportion of different surgical methods performed on patients with astrocytoma between 2004 and 2015. EB, excision biopsy; STR, subtotal resection; GR, gross resection; PR, partial resection; GTR, gross total resection.

and chemotherapy information, Cox regression indicated that compared with EB patients, the non-surgical patients [hazard ratio (HR), 1.45; 95\% confidence interval (CI), 1.41-1.50; $\mathrm{P}<0.001)$ and patients with PR (HR, 1.04; 95\% CI, 1.00-1.08; $\mathrm{P}=0.038)$ had less favorable $\mathrm{OS}$, whereas patients with $\mathrm{GR}$ (HR, 0.72; 95\% CI, 0.69-0.75; P<0.001) and GTR (HR, 0.80; 95\% CI, 0.77-0.83; P<0.001) had more favorable OS. In terms of CSS, compared with other patients, the non-surgical patients (vs. EB; HR, 1.43; 95\% CI, 1.38-1.49; P<0.001) had significantly lower odds of CSS, whereas GR patients (vs. EB; HR, 0.72; 95\% CI, 0.69-0.76; $\mathrm{P}<0.001$ ) and GTR patients (vs. EB; HR, 0.80; 95\% CI, 0.77-0.83; P<0.001) had significantly greater odds of CSS. Surgical method was an independent prognostic factor for OS and CSS in patients with astrocytoma.

Subgroup analysis for evaluating the effect of surgical method on OS and CSS based on summary stage and WHO grade.
Based on summary stage and WHO grade, the difference between surgical method and prognosis among the subgroups of astrocytoma patients was further examined (Table IV). It was found that for OS and CSS, surgical method was still an independent prognostic factor for patients with localized, regional, distant, grade II, grade III and grade IV. Compared with patients with EB, patients with GTR in the localized group (OS: HR, 0.82; 95\% CI, 0.79-0.86; P<0.001; CSS: HR, 0.82, 95\% CI, 0.79-0.86; $\mathrm{P}<0.001$ ), regional group (OS: HR, 0.68; 95\% CI, 0.61-0.76; P<0.001; CSS: HR, 0.67; 95\% CI, 0.59-0.75; $\mathrm{P}<0.001$ ), distant group (OS: HR, 0.75; 95\% CI, 0.53-1.06; P<0.001; CSS: H, R0.69; 95\% CI, 0.47-1.00; $\mathrm{P}=0.051$ ), grade II group (OS: HR, 0.77; 95\% CI, 0.66-0.90; $\mathrm{P}<0.001$; CSS: HR, 0.78; 95\% CI, 0.66-0.92; $\mathrm{P}<0.001)$, grade III group (OS: HR, 0.57; 95\% CI, 0.48-0.68; P<0.001; CSS: HR, 0.57; 95\% CI, 0.48-0.69; $\mathrm{P}<0.001)$ and grade IV group (OS: HR, 0.82; 95\% CI, 0.79-0.87; P<0.001; CSS: HR, 0.83; 95\% CI, 0.79-0.86; $\mathrm{P}<0.001$ ) had higher relative survival rates. Moreover, in the grade II and III subgroups, GTR was associated with the highest OS and CSS MST for patients. However, for patients in the grade I subgroup, the multivariate Cox regression showed that the surgical method had no significant effect on OS or CSS ( $\mathrm{P}>0.05)$. The subtype stratification based on summary stage and WHO grade is graphically displayed in Figs. 5 and 6, respectively.

\section{Discussion}

The present study used a large, population-based database to quantitatively compare the impact of four different surgical methods on survival in patients with astrocytoma. The effect of surgical method on OS and CSS rate in patients with astrocytoma was analyzed and it was found that surgical method was an independent prognostic factor for patients with astrocytoma. Patients in the GR and GTR groups had higher OS and CSS time MST compared with those in the non-surgical, EB and PR groups and similar results were obtained in subgroup analyses based on summary stage and grade. However, although GR and GTR had higher OS and CSS time, it was 
Table II. Kaplan-Meier analysis overall survival and cancer-specific survival for patients with astrocytoma.

\begin{tabular}{|c|c|c|c|c|c|c|}
\hline \multirow[b]{2}{*}{ Characteristic } & \multirow[b]{2}{*}{ OS MST, months } & \multicolumn{2}{|c|}{ Kaplan-Meier } & \multirow[b]{2}{*}{ CSS MST, months } & \multicolumn{2}{|c|}{ Kaplan-Meier } \\
\hline & & Log-rank test & P-value & & Log-rank test & P-value \\
\hline Age at diagnosis, years & & $12,929.451$ & $<0.001$ & & $10,171.401$ & $<0.001$ \\
\hline $18-40$ & 77.00 & & & 112.00 & & \\
\hline $41-60$ & 15.00 & & & 17.00 & & \\
\hline $61-80$ & 6.00 & & & 8.00 & & \\
\hline$>60$ & 2.00 & & & 3.00 & & \\
\hline Sex & & 2.209 & 0.137 & & 4.125 & 0.042 \\
\hline Male & 10.00 & & & 13.00 & & \\
\hline Female & 10.00 & & & 12.00 & & \\
\hline Ethnicity & & 116.719 & $<0.001$ & & 143.124 & $<0.001$ \\
\hline White & 10.00 & & & 12.00 & & \\
\hline Black & 11.00 & & & 15.00 & & \\
\hline Other & 14.00 & & & 18.00 & & \\
\hline Marital status & & 7.893 & 0.019 & & 6.171 & 0.046 \\
\hline Married & 11.00 & & & 13.00 & & \\
\hline Unmarried & 8.00 & & & 11.00 & & \\
\hline Unknown & 10.00 & & & 13.00 & & \\
\hline Urban-rural residence & & 87.138 & $<0.001$ & & 72.102 & $<0.001$ \\
\hline Metropolitan & 11.00 & & & 8.00 & & \\
\hline Non-metropolitan & 8.00 & & & 6.00 & & \\
\hline Income & & 122.487 & $<0.001$ & & 82.768 & $<0.001$ \\
\hline Lower & 9.00 & & & 11.00 & & \\
\hline Middle & 10.00 & & & 12.00 & & \\
\hline Upper & 11.00 & & & 14.00 & & \\
\hline Summary stage & & $1,208.088$ & $<0.001$ & & $1,097.821$ & $<0.001$ \\
\hline Localized & 12.00 & & & 14.00 & & \\
\hline Regional & 6.00 & & & 7.00 & & \\
\hline Distant & 4.00 & & & 5.00 & & \\
\hline Unstaged/unknown & 5.00 & & & 8.00 & & \\
\hline WHO grade & & $6,264.594$ & $<0.001$ & & $5,846.311$ & $<0.001$ \\
\hline $\mathrm{I}$ & - & & & - & & \\
\hline II & 39.00 & & & 66.00 & & \\
\hline III & 20.00 & & & 25.00 & & \\
\hline IV & 8.00 & & & 10.00 & & \\
\hline Surgery & & 3212.688 & $<0.001$ & & $2,281.789$ & $<0.001$ \\
\hline No surgery & 4.00 & & & 5.00 & & \\
\hline $\mathrm{EB}$ & 11.00 & & & 13.00 & & \\
\hline STR & 12.00 & & & 14.00 & & \\
\hline GR & 17.00 & & & 19.00 & & \\
\hline PR & 10.00 & & & 12.00 & & \\
\hline GTR & 15.00 & & & 17.00 & & \\
\hline Radiotherapy & & $1,377.534$ & $<0.001$ & & 712.575 & $<0.001$ \\
\hline Yes & 13.00 & & & 15.00 & & \\
\hline No & 3.00 & & & 4.00 & & \\
\hline Chemotherapy & & $1,392.837$ & $<0.001$ & & 697.593 & $<0.001$ \\
\hline Yes & 14.00 & & & 16.00 & & \\
\hline No & 3.00 & & & 5.00 & & \\
\hline
\end{tabular}

MST, median survival time; OS, overall survival; CSS, cause-specific survival; EB, excision biopsy; STR, subtotal resection; GR, gross resection; PR, partial resection; GTR, gross total resection. 

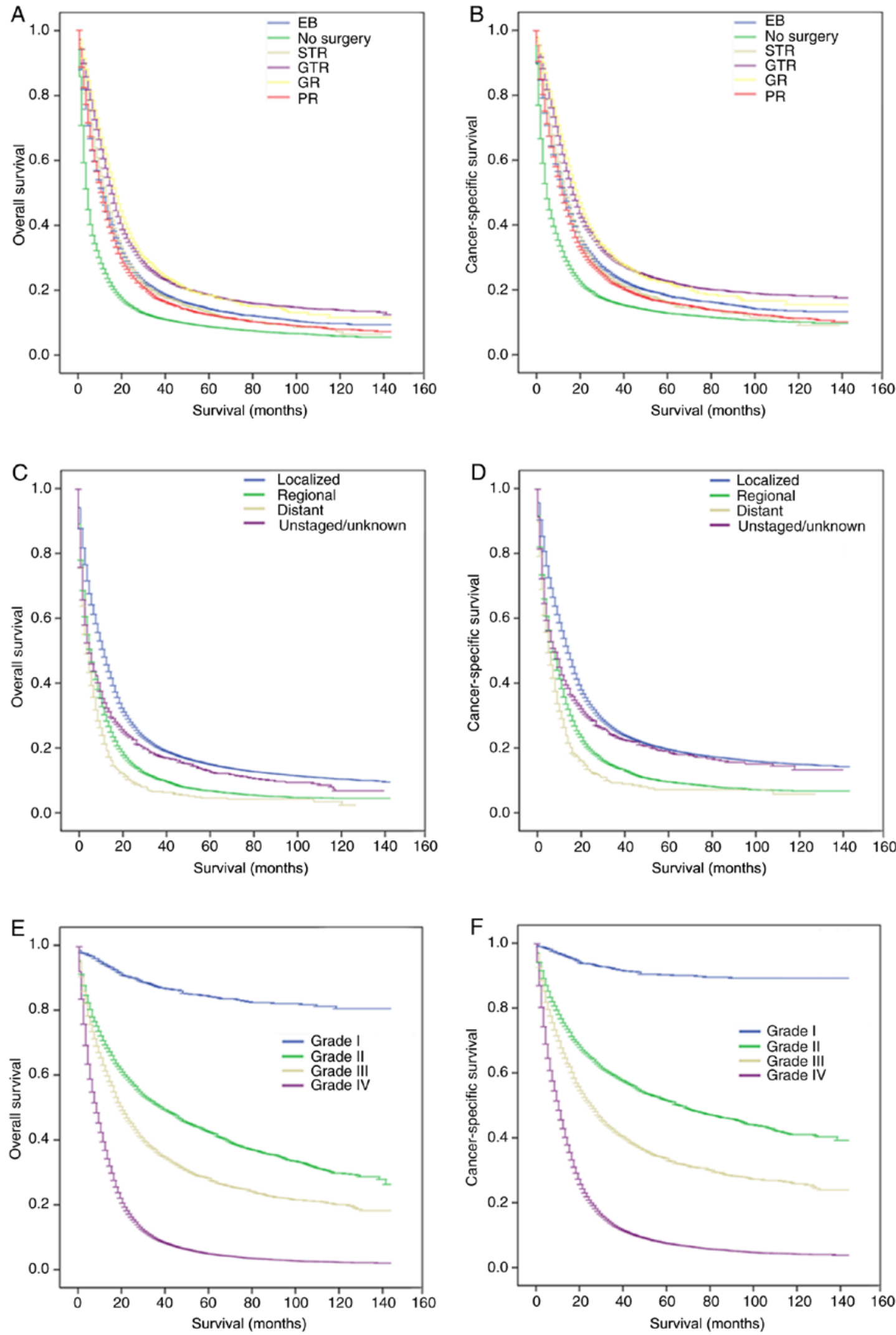

Figure 4. Kaplan-Meier survival curves according to surgical method, summary stage and WHO grade (7) in patients with astrocytoma. (A and B) Overall survival (left) and cancer-specific survival (right) based on surgical method. (C and D) Overall survival (left) and cancer-specific survival (right) based on summary stage. (E and F) Overall survival (left) and cancer-specific survival (right) based on WHO grade. EB, excision biopsy; STR, subtotal resection; GR, gross resection; PR, partial resection; GTR, gross total resection; WHO, World Health Organization.

observed that the percentage of patients who underwent GTR decreased between 2004 and 2015 .
Surgical resection serves a key role in the management of patients with grade I, II and III astrocytoma (18). 
Table III. Risk factors for overall survival and cancer-specific survival for patients with astrocytoma.

\begin{tabular}{|c|c|c|c|c|}
\hline \multirow[b]{2}{*}{ Characteristic } & \multicolumn{2}{|c|}{ OS } & \multicolumn{2}{|c|}{ CSS } \\
\hline & $\mathrm{HR}(95 \% \mathrm{CI})$ & P-value & $\operatorname{HR}(95 \% \mathrm{CI})$ & P-value \\
\hline \multicolumn{5}{|l|}{ Age at diagnosis, years } \\
\hline $18-40$ & Reference & & Reference & \\
\hline $41-60$ & $2.29(2.18-2.41)$ & $<0.001$ & $2.24(2.12-2.36)$ & $<0.001$ \\
\hline $61-80$ & $3.85(3.66-4.05)$ & $<0.001$ & $3.66(3.47-3.86)$ & $<0.001$ \\
\hline$>60$ & $5.85(5.51-6.20)$ & $<0.001$ & $5.47(5.13-5.84)$ & $<0.001$ \\
\hline \multicolumn{5}{|l|}{ Ethnicity } \\
\hline White & Reference & & Reference & \\
\hline Black & $0.92(0.88-0.97)$ & 0.001 & $0.87(0.82-0.91)$ & $<0.001$ \\
\hline Other & $0.84(0.80-0.88)$ & $<0.001$ & $0.82(0.77-0.86)$ & $<0.001$ \\
\hline \multicolumn{5}{|l|}{ Marital status } \\
\hline Married & Reference & & Reference & \\
\hline Unmarried & $1.10(1.07-1.12)$ & $<0.001$ & $1.08(1.05-1.10)$ & $<0.001$ \\
\hline Unknown & $0.96(0.91-1.02)$ & 0.179 & $0.92(0.86-0.98)$ & 0.009 \\
\hline \multicolumn{5}{|l|}{ Urban-rural residence } \\
\hline Metropolitan & Reference & & Reference & \\
\hline Non-metropolitan & $1.05(1.01-1.09)$ & 0.015 & $1.06(1.02-1.11)$ & 0.003 \\
\hline \multicolumn{5}{|l|}{ Income } \\
\hline Lower & Reference & & Reference & \\
\hline Middle & $0.94(0.92-0.97)$ & $<0.001$ & $0.97(0.94-1.00)$ & 0.055 \\
\hline Upper & $0.88(0.85-0.91)$ & $<0.001$ & $0.89(0.86-0.92)$ & $<0.001$ \\
\hline \multicolumn{5}{|l|}{ Summary stage } \\
\hline Localized & Reference & & Reference & \\
\hline Regional & $1.43(1.39-1.47)$ & $<0.001$ & $1.46(1.41-1.50)$ & $<0.001$ \\
\hline Distant & $1.49(1.36-1.62)$ & $<0.001$ & $1.53(1.40-1.69)$ & $<0.001$ \\
\hline Unstaged/unknown & $0.75(0.70-0.80)$ & $<0.001$ & $0.75(0.70-0.81)$ & $<0.001$ \\
\hline \multicolumn{5}{|l|}{ WHO grade } \\
\hline I & Reference & & Reference & \\
\hline II & $5.01(4.23-5.95)$ & $<0.001$ & $6.49(5.21-8.09)$ & $<0.001$ \\
\hline III & $10.09(8.50-11.98)$ & $<0.001$ & $14.23(11.40-17.75)$ & $<0.001$ \\
\hline IV & $18.34(15.50-21.70)$ & $<0.001$ & $26.39(21.22-32.82)$ & $<0.001$ \\
\hline \multicolumn{5}{|l|}{ Surgery } \\
\hline No surgery & $1.45(1.41-1.50)$ & $<0.001$ & $1.43(1.38-1.49)$ & $<0.001$ \\
\hline EB & Reference & & Reference & \\
\hline STR & $0.95(0.92-0.99)$ & 0.022 & $0.96(0.92-1.00)$ & 0.068 \\
\hline GR & $0.72(0.69-0.75)$ & $<0.001$ & $0.72(0.69-0.76)$ & $<0.001$ \\
\hline PR & $1.04(1.00-1.08)$ & 0.038 & $1.04(1.00-1.08)$ & 0.069 \\
\hline GTR & $0.80(0.77-0.83)$ & $<0.001$ & $0.80(0.77-0.83)$ & $<0.001$ \\
\hline \multicolumn{5}{|l|}{ Radiotherapy } \\
\hline Yes & Reference & & Reference & \\
\hline No & $1.61(1.56-1.67)$ & $<0.001$ & $1.58(1.52-1.64)$ & $<0.001$ \\
\hline \multicolumn{5}{|l|}{ Chemotherapy } \\
\hline Yes & Reference & & Reference & \\
\hline No & $1.55(1.50-1.60)$ & $<0.001$ & $1.49(1.45-1.55)$ & $<0.001$ \\
\hline
\end{tabular}

HR, hazard ratio; CI, confidence interval; OS, overall survival; CSS, cause-specific survival; EB, excision biopsy; GR, gross resection; PR, partial resection; STR, subtotal resection; GTR, gross total resection.

For patients with grade I astrocytoma, surgical resection is usually effective and these patients rarely receive radiotherapy and chemotherapy (19). Johnson et al (20) retrospectively analyzed 865 adult patients with pilocytic astrocytoma aged 
Table IV. Subgroup analyses stratified by summary stage and grade for overall survival and cancer-specific survival for patients with.

\begin{tabular}{|c|c|c|c|c|c|c|}
\hline \multirow[b]{2}{*}{ Characteristic } & \multirow[b]{2}{*}{ OS MST, months } & \multicolumn{2}{|l|}{$\mathrm{OS}$} & \multirow[b]{2}{*}{ CSS MST, months } & \multicolumn{2}{|l|}{ CSS } \\
\hline & & $\mathrm{HR}(95 \% \mathrm{CI})$ & P-value & & $\operatorname{HR}(95 \% \mathrm{CI})$ & P-value \\
\hline \multicolumn{7}{|l|}{ Localized } \\
\hline No surgery & 4.00 & $1.54(1.48-1.60)$ & $<0.001$ & 6.00 & $1.51(1.44-1.57)$ & $<0.001$ \\
\hline EB & 12.00 & Reference & - & 15.00 & Reference & - \\
\hline STR & 13.00 & $0.99(0.94-1.04)$ & 0.611 & 15.00 & $1.00(0.96-1.05)$ & 0.879 \\
\hline GR & 17.00 & $0.73(0.70-0.77)$ & $<0.001$ & 19.00 & $0.74(0.70-0.77)$ & $<0.001$ \\
\hline PR & 11.00 & $1.07(1.02-1.12)$ & 0.002 & 13.00 & $1.07(1.02-1.12)$ & 0.007 \\
\hline GTR & 16.00 & $0.82(0.79-0.86)$ & $<0.001$ & 14.00 & $0.82(0.79-0.86)$ & $<0.001$ \\
\hline \multicolumn{7}{|l|}{ Regional } \\
\hline No surgery & 3.00 & $1.31(1.22-1.41)$ & $<0.001$ & 4.00 & $1.30(1.20-1.40)$ & $<0.001$ \\
\hline EB & 7.00 & Reference & - & 8.00 & Reference & - \\
\hline STR & 10.00 & $0.85(0.78-0.94)$ & 0.001 & 11.00 & $0.83(0.75-0.92)$ & $<0.001$ \\
\hline GR & 12.00 & $0.70(0.63-0.79)$ & $<0.001$ & 13.00 & $0.71(0.62-0.80)$ & $<0.001$ \\
\hline PR & 8.00 & $0.94(0.86-1.03)$ & 0.175 & 9.00 & $0.95(0.86-1.04)$ & 0.277 \\
\hline GTR & 11.00 & $0.68(0.61-0.76)$ & $<0.001$ & 13.00 & $0.67(0.59-0.75)$ & $<0.001$ \\
\hline \multicolumn{7}{|l|}{ Distant } \\
\hline No surgery & 2.00 & $1.21(0.94-1.55)$ & 0.133 & 2300 & $1.20(0.92-1.56)$ & 0.177 \\
\hline EB & 5.00 & Reference & - & 5.00 & Reference & - \\
\hline STR & 8.00 & $0.79(0.58-1.07)$ & 0.131 & 10.00 & $0.72(0.52-1.01)$ & 0.056 \\
\hline GR & 9.00 & $0.65(0.45-0.94)$ & 0.023 & 13.00 & $0.58(0.39-0.88)$ & 0.010 \\
\hline PR & 5.00 & $0.68(0.49-0.94)$ & 0.021 & 7.00 & $0.63(0.44-0.90)$ & 0.012 \\
\hline GTR & 6.00 & $0.75(0.53-1.06)$ & 0.099 & 7.00 & $0.69(0.47-1.00)$ & 0.051 \\
\hline \multicolumn{7}{|l|}{ Grade I } \\
\hline No surgery & & NA & 0.112 & & NA & 0.169 \\
\hline $\mathrm{EB}$ & - & Reference & - & & Reference & - \\
\hline STR & - & NA & 0.564 & & NA & 0.388 \\
\hline GR & - & NA & 0.823 & & NA & 0.706 \\
\hline PR & - & NA & 0.724 & & NA & 0.356 \\
\hline GTR & - & NA & 0.885 & & NA & 0.310 \\
\hline \multicolumn{7}{|l|}{ Grade II } \\
\hline No surgery & 18.00 & $1.26(1.13-1.40)$ & $<0.001$ & 31.00 & $1.17(1.04-1.32)$ & 0.012 \\
\hline EB & 44.00 & Reference & - & 69.00 & Reference & - \\
\hline STR & 49.00 & $0.88(0.73-1.06)$ & 0.063 & 63.00 & $0.86(0.70-1.05)$ & 0.138 \\
\hline GR & 96.00 & $0.71(0.58-0.86)$ & $<0.001$ & - & $0.71(0.57-0.88)$ & 0.002 \\
\hline PR & 39.00 & $1.14(0.99-1.31)$ & 0.062 & 64.00 & $1.05(0.90-1.23)$ & 0.516 \\
\hline GTR & 104.00 & $0.77(0.66-0.90)$ & 0.001 & - & $0.78(0.66-0.92)$ & 0.004 \\
\hline \multicolumn{7}{|l|}{ Grade III } \\
\hline No surgery & 8.00 & $1.45(1.30-1.62)$ & $<0.001$ & 10.00 & $1.51(1.34-1.69)$ & $<0.001$ \\
\hline $\mathrm{EB}$ & 20.00 & Reference & - & 24.00 & Reference & - \\
\hline STR & 35.00 & $0.85(0.72-1.01)$ & 0.064 & 47.00 & $0.83(0.69-1.00)$ & 0.050 \\
\hline GR & 72.00 & $0.55(0.45-0.67)$ & $<0.001$ & 75.00 & $0.55(0.45-0.68)$ & $<0.001$ \\
\hline PR & 29.00 & $1.02(0.87-1.18)$ & 0.836 & 33.00 & $1.02(0.87-1.20)$ & 0.809 \\
\hline GTR & 78.00 & $0.57(0.48-0.68)$ & $<0.001$ & 119.00 & $0.57(0.48-0.69)$ & $<0.001$ \\
\hline \multicolumn{7}{|l|}{ Grade IV } \\
\hline No surgery & 3.00 & $1.45(1.40-1.51)$ & $<0.001$ & 4.00 & $1.42(1.37-1.48)$ & $<0.001$ \\
\hline EB & 9.00 & Reference & - & 10.00 & Reference & - \\
\hline STR & 10.00 & $0.98(0.94-1.02)$ & 0.339 & 11.00 & $0.99(0.95-1.04)$ & 0.703 \\
\hline GR & 14.00 & $0.74(0.71-0.77)$ & $<0.001$ & 16.00 & $0.75(0.710 .78)$ & $<0.001$ \\
\hline PR & 9.00 & $1.03(0.99-1.08)$ & 0.138 & 10.00 & $1.04(0.99-1.08)$ & 0.116 \\
\hline GTR & 13.00 & $0.82(0.79-0.87)$ & $<0.001$ & 14.00 & $0.83(0.79-0.86)$ & $<0.001$ \\
\hline
\end{tabular}

HR, hazard ratio; CI, confidence interval; OS, overall survival; CSS, cause-specific survival; EB, excision biopsy; STR, subtotal resection; GR, gross resection; PR, partial resection; GTR, gross total resection. 

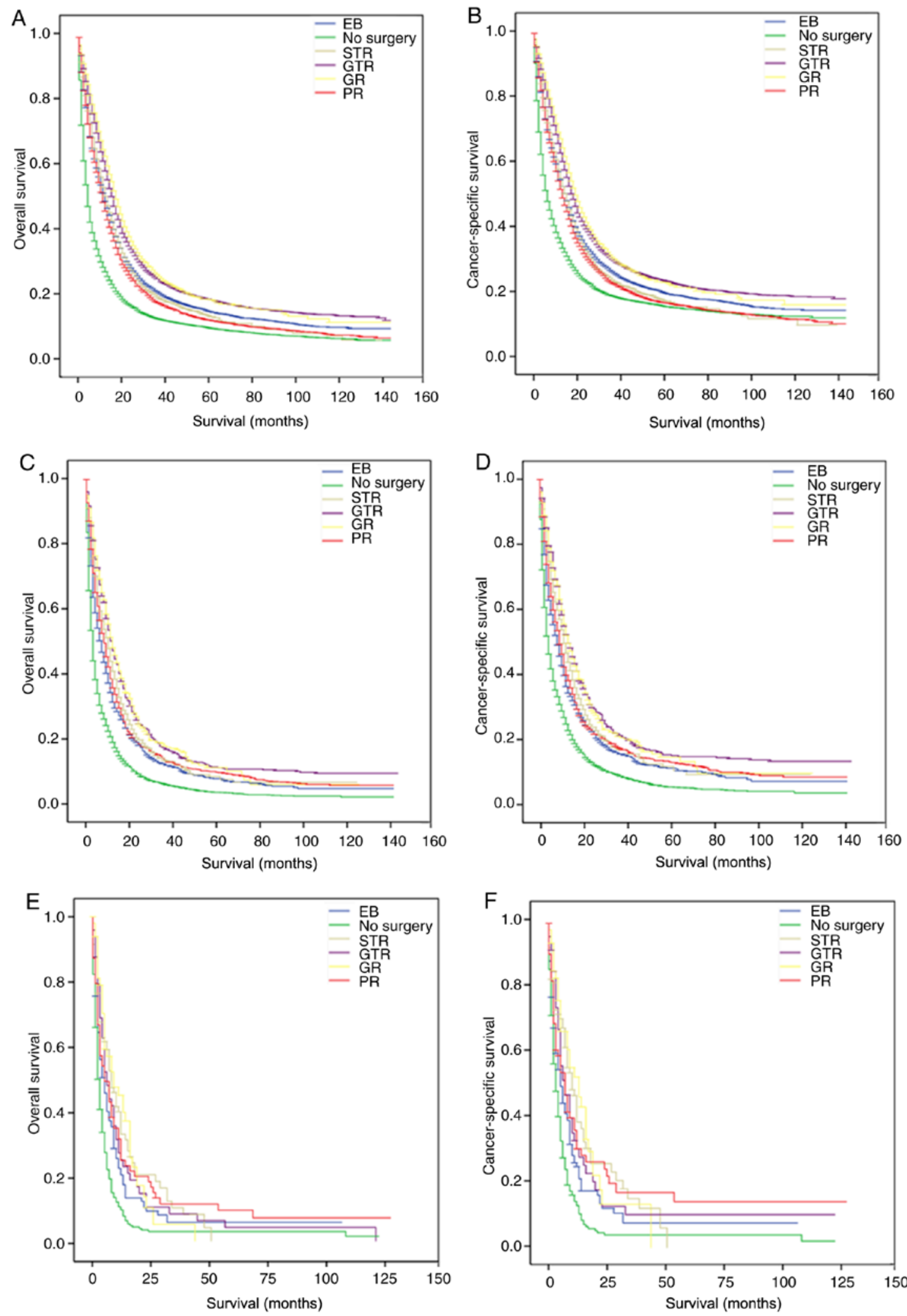

Figure 5. OS and CSS curves of patients with astrocytoma according to the surgical method undergone at different summary stages. (A and B) OS (left) and CSS (right) in patients with localized summary stage. (C and D) OS (left) and CSS (right) in patients with regional summary stage. (E and F) OS (left) and CSS (right) in patients with distant summary stage. EB, excision biopsy; STR, subtotal resection; GR, gross resection; PR, partial resection; GTR, gross total resection; OS, overall survival; CSS, cancer-specific survival.

20 years and older, and reported that GTR was a significant predictor of survival compared with STR or biopsy. Several studies have shown that the extent of resection can affect the OS of patients with grade II and III astrocytoma (21-23). Fouladi et al (24) found that for patients with pleomorphic xanthoastrocytoma, GTR without adjuvant therapy prolonged 

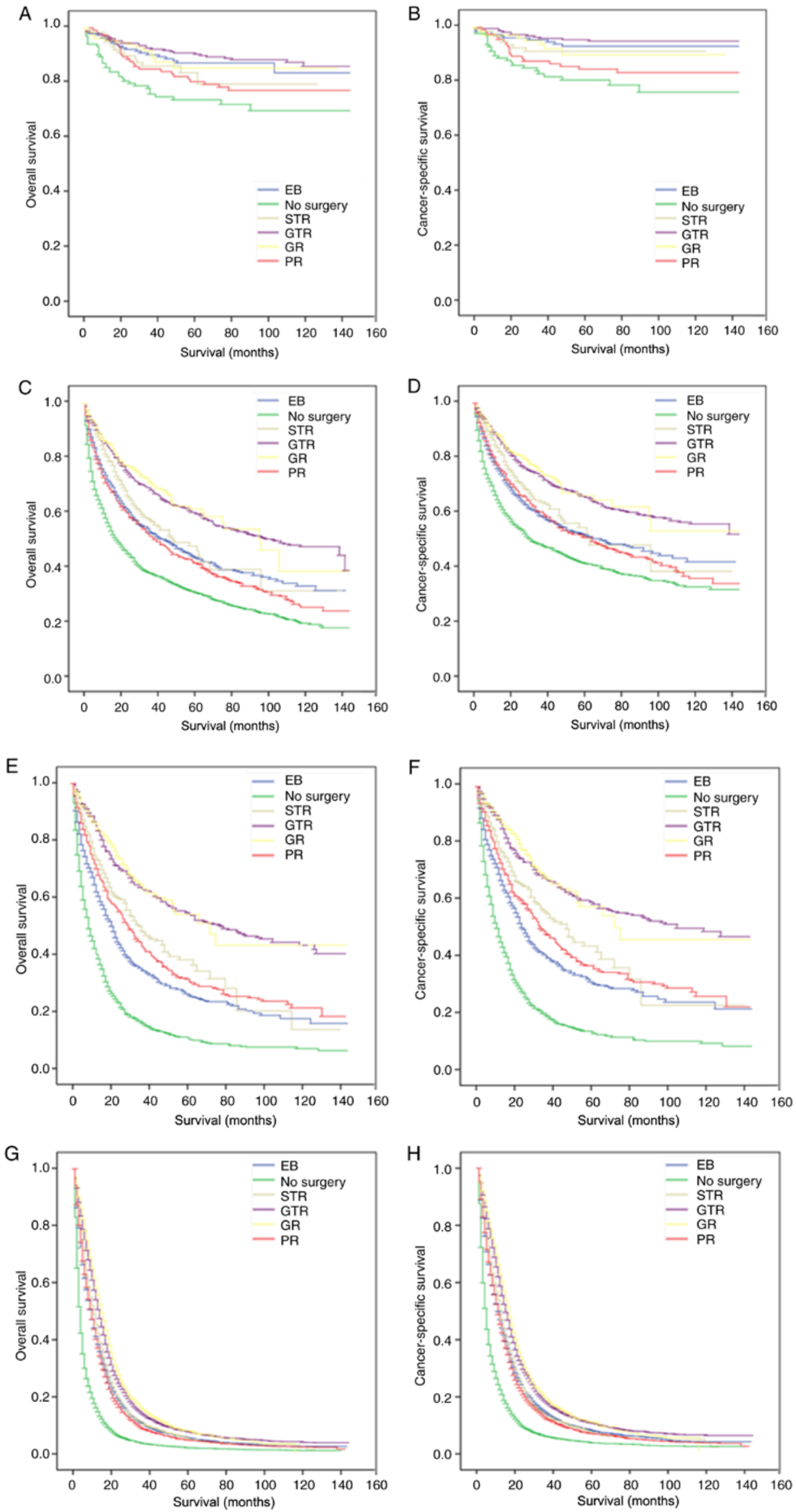

Figure 6. Overall survival and cancer-specific survival curves of patients with astrocytoma patients according to the surgical method undergone in different World Health organization grades (7). (A and B) OS (left) and CSS (right) in patients with grade I. (C and D) OS (left) and CSS (right) in patients with grade II. (E and F) OS (left) and CSS (right) in patients with grade III. (G and H) OS (left) and CSS (right) in patients with grade IV. EB, excision biopsy; STR, subtotal resection; GR, gross resection; PR, partial resection; GTR, gross total resection; OS, overall survival; CSS, cancer-specific survival. 
disease control. Moreover, for patients with glioblastoma (GBM), there is evidence to support the benefit of GTR with regard to survival $(25,26)$. A large single-center study based on 1,229 patients with GBM showed that GTR significantly prolonged MST compared with incomplete resection (27).

In the present study, statistical analysis of all patients with astrocytoma was performed, demonstrating that GTR and GR were beneficial for the survival of astrocytoma patients and could reduce the risk of death. Subsequently, a stratified analysis based on the summary stage and WHO grade was conducted and showed that GTR was beneficial for OS and CSS. For patients with localized, regional and distant astrocytoma, GR was associated with the longest OS and CSS time MST, whereas GTR was associated with similar survival times and benefits. In the stratified analysis according to WHO stage, the benefits of GTR were more prominent compared with other analyses, and GTR could lead to the longest OS and CSS time MST in patients with grade II and III.

Maximizing the benefit of resection is a core principle of neurosurgical oncology and every effort should be made to achieve GTR during the initial surgery. The present study observed that the proportion of patients who received STR increased from 2004 to 2015 . This may be since studies have shown that patients who underwent STR of thalamic and brain stem gliomas had a relatively good prognosis $(28,29)$. Minehan et al (30) studied 136 patients with spinal astrocytoma and found that 11 patients with GTR had the shortest median survival time, which may be the reason for the decrease in the proportion of patients with GTR.

In the present study of patients with astrocytoma, the mortality rate of patients treated with PR was higher compared with that of patients receiving EB, STR, GR or GTR. After further stratified analysis of the summary stage, it was observed that this effect gradually weakened with increased stage. For patients with distant summary stage, PR was more beneficial compared with GTR. This phenomenon was further analyzed and it was indicated that this may be associated with the fact that PR produces a smaller residual tumour volume compared with EB, which can slow the tumour growth rate. In addition, GTR is more traumatic for the patient compared with PR (31). Total surgical resection should be considered with caution by the surgeon, as it is strictly dependent on the anatomical location of the tumour, as well as the presence of patient comorbidities (17). Therefore, for different patients with astrocytoma, different and individual treatments are necessary.

There are limitations to the present study. Firstly, the SEER database is a retrospective dataset with its own retrospective study limitations. Secondly, the patients' physical conditions were unclear and patients with several comorbidities may pursue more conservative treatment. Thirdly, there may be selection bias with patients receiving GTR compared with STR. This may be due to the surgeons who would consider the postoperative complications of GTR surgery for astrocytoma patients. In addition, for chemotherapy and radiotherapy, the present study does not distinguish whether adjuvant or neoadjuvant therapy was used and there is no information on the specific radiotherapy technique, including dose, fractionation and beam energy, or chemotherapy regimen used.
In the present study, it was demonstrated that the survival benefit of GTR was higher compared with unsuccessful or not attempted GTR, therefore more patients need to be encouraged to undergo GTR to improve OS and CSS times.

\section{Acknowledgements}

Not applicable.

\section{Funding}

No funding was received.

\section{Availability of data and material}

The datasets generated and/or analyzed during the present study are available in the Surveillance, Epidemiology, and End Results Program repository (seer.cancer.gov/).

\section{Authors' contributions}

HM and XL were involved in the study conception and design. HM collected and assembled data. HM and WM were involved in data analysis and interpretation. HM and XL wrote the manuscript. All authors read and approved the final manuscript.

\section{Ethics approval and consent to participate}

No applicable.

\section{Patient consent for publication}

No applicable.

\section{Competing interests}

The authors declare that they have no competing interests.

\section{References}

1. Teng YD,Abd-El-Barr M, Wang L,Hajiali H,WuLandZafonteRD: Spinal cord astrocytomas: Progresses in experimental and clinical investigations for developing recovery neurobiology-based novel therapies. Exp Neurol 311: 135-147, 2019.

2. Iida T, Tomogane Y, Takagi T, Miyaji Y, Sakamoto D, Yoshida Y, Ishikura R, Ando K, Nakagomi N, Hirota S and Yoshimura S: Grading of astrocytomas using the PRESTO (principles of echo-shifting with a train of observations) magnetic resonance imaging sequence. Clin Neurol Neurosurg 173: 91-95, 2018.

3. Xie JC, Yang S, Liu XY and Zhao YX: Marital status is associated with survival of patients with astrocytoma. J Clin Neurosci 56: 79-87, 2018.

4. Wen PY and Kesari S: Malignant gliomas in adults. N Engl J Med 359: 492-507, 2008.

5. Ostrom QT, Cioffi G, Gittleman H, Patil N, Waite K, Kruchko C and Barnholtz-Sloan JS: CBTRUS statistical report: Primary brain and other central nervous system tumors diagnosed in the United States in 2012-2016. Neuro Oncol 21 (Suppl 5): v1-v100, 2019.

6. Walker DG and Kaye AH: Diagnosis and management of astrocytomas, oligodendrogliomas and mixed gliomas: A review. Australas Radiol 45: 472-482, 2001.

7. Donofrio CA, Gagliardi F, Callea M, da Passano CF, Terreni MR, Cavalli A, Spina A, Acerno S, Bailo M, Elbabaa SK and Mortini P: Pediatric cerebellar pilocytic astrocytoma presenting with spontaneous intratumoral hemorrhage. Neurosurg Rev 2018 (Epub ahead of print). 
8. Louis DN, Perry A, Reifenberger G, von Deimling A, Figarella-Branger D, Cavenee WK, Ohgaki H, Wiestler OD, Kleihues P and Ellison DW: The 2016 world health organization classification of tumors of the central nervous system: A summary. Acta Neuropathol 131: 803-820, 2016

9. Weidmann MJ: Neurosurgery. Med J Aust 161: 392-394, 1994.

10. Tavares CB, Gomes-Braga FDCS, Sousa EB, Borges US, Escórcio-Dourado CS, Silva-Sampaio JPD and Silva BBD: Evaluation of estrogen receptor expression in low-grade and high-grade astrocytomas. Rev Assoc Med Bras (1992) 64 1129-1133, 2018

11. Guastella AR, Michelhaugh SK, Klinger NV, Fadel HA, Kiousis S, Ali-Fehmi R, Kupsky WJ, Juhász C and Mittal S: Investigation of the aryl hydrocarbon receptor and the intrinsic tumoral component of the kynurenine pathway of tryptophan metabolism in primary brain tumors. J Neurooncol 139: 239-249, 2018.

12. Delgado-Lopez PD, Corrales-Garcia EM, Martino J, Lastra-Aras E and Duenas-Polo MT: Diffuse low-grade glioma: A review on the new molecular classification, natural history and current management strategies. Clin Transl Oncol 19: 931-944, 2017.

13. Patel S, DiBiase S, Meisenberg B, Flannery T, Patel A, Dhople A, Cheston S and Amin P: Phase I clinical trial assessing temozolomide and tamoxifen with concomitant radiotherapy for treatment of high-grade glioma. Int J Radiat Oncol Biol Phys 82: 739-742, 2012.

14. Ghotme KA, Barreto GE, Echeverria V, Gonzalez J, Bustos RH, Sanchez M, Leszek J, Yarla NS, Gomez RM, Tarasov VV, et al: Gliomas: New perspectives in diagnosis, treatment and prognosis. Curr Top Med Chem 17: 1438-1447, 2017.

15. Mao W, Huang X, Kong M, Fan J and Geng J: More lymph node dissection improves survival in patients with newly diagnosed lymph node-positive penile cancer. Int Urol Nephrol 51: 641-654, 2019.

16. Mao W, Kong M, Yu H, Wang D, Huang X, Yao X, Fan J and Geng J: Prognosis and treatment differences between initial and second primary chondrosarcoma. Oncol Lett 18: 207-218, 2019.

17. Alattar AA, Brandel MG, Hirshman BR, Dong X, Carroll KT, Ali MA, Carter BS and Chen CC: Oligodendroglioma resection: A surveillance, epidemiology, and end results (SEER) analysis. J Neurosurg 128: 1076-1083, 2018.

18. Dong X, Noorbakhsh A, Hirshman BR, Zhou T, Tang JA, Chang DC, Carter BS and Chen CC: Survival trends of grade I, II, and III astrocytoma patients and associated clinical practice patterns between 1999 and 2010: A SEER-based analysis. Neurooncol Pract 3: 29-38, 2016.

19. Karajannis M, Allen JC and Newcomb EW: Treatment of pediatric brain tumors. J Cell Physiol 217: 584-589, 2008.

20. Johnson DR, Brown PD, Galanis E and Hammack JE: Pilocytic astrocytoma survival in adults: Analysis of the surveillance, epidemiology, and end results program of the national cancer institute. J Neurooncol 108: 187-193, 2012.
21. Keles GE, Chang EF, Lamborn KR, Tihan T, Chang CJ, Chang SM and Berger MS: Volumetric extent of resection and residual contrast enhancement on initial surgery as predictors of outcome in adult patients with hemispheric anaplastic astrocytoma. J Neurosurg 105: 34-40, 2006.

22. Smith JS, Chang EF, Lamborn KR, Chang SM, Prados MD, Cha S, Tihan T, Vandenberg S, McDermott MW and Berger MS: Role of extent of resection in the long-term outcome of low-grade hemispheric gliomas. J Clin Oncol 26: 1338-1345, 2008.

23. McGirt MJ, Chaichana KL, Attenello FJ, Weingart JD, Than K, Burger PC, Olivi A, Brem H and Quinoñes-Hinojosa A: Extent of surgical resection is independently associated with survival in patients with hemispheric infiltrating low-grade gliomas. Neurosurgery 63: 700-707, 2008.

24. Fouladi M, Jenkins J, Burger P, Langston J, Merchant T, Heideman R, Thompson S, Sanford A, Kun L and Gajjar A: Pleomorphic xanthoastrocytoma: Favorable outcome after complete surgical resection. Neuro Oncol 3: 184-192, 2001.

25. Lacroix M, Abi-Said D, Fourney DR, Gokaslan ZL, Shi W, DeMonte F, Lang FF, McCutcheon IE, Hassenbusch SJ, Holland E, et al: A multivariate analysis of 416 patients with glioblastoma multiforme: Prognosis, extent of resection, and survival. J Neurosurg 95: 190-198, 2001.

26. Sanai N and Berger MS: Glioma extent of resection and its impact on patient outcome. Neurosurgery 62: 753-764; discussion 264-756, 2008

27. Li YM, Suki D, Hess K and Sawaya R: The influence of maximum safe resection of glioblastoma on survival in 1229 patients: Can we do better than gross-total resection? J Neurosurg 124: 977-988, 2016.

28. Freeman CR and Suissa S: Brain stem tumors in children: Results of a survey of 62 patients treated with radiotherapy. Int J Radiat Oncol Biol Phys 12: 1823-1828, 1986.

29. Grigsby PW, Thomas PR, Schwartz HG and Fineberg BB Multivariate analysis of prognostic factors in pediatric and adult thalamic and brainstem tumors. Int J Radiat Oncol Biol Phys 16: 649-655, 1989 .

30. Minehan KJ, Brown PD, Scheithauer BW, Krauss WE and Wright MP: Prognosis and treatment of spinal cord astrocytoma. Int J Radiat Oncol Biol Phys 73: 727-733, 2009.

31. Hongo H, Takai K, Komori T and Taniguchi M: Intramedullary spinal cord ependymoma and astrocytoma: Intraoperative frozen-section diagnosis, extent of resection, and outcomes. J Neurosurg Spine 30: 133-139, 2019.

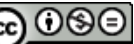

This work is licensed under a Creative Commons Attribution-NonCommercial-NoDerivatives 4.0 International (CC BY-NC-ND 4.0) License. 